\title{
Latent Celiac Disease
}

National Cancer Institute

\section{Source}

National Cancer Institute. Latent Celiac Disease. NCI Thesaurus. Code C45425.

Diagnosis of celiac disease at a later age despite an asymptomatic clinical course.

Serologic evidence with minimal histological changes in the intestinal mucosa are noted.

The presence of human tissue transglutaminase, endomysial and gliadin antibodies

without substantial villous changes follow many years of a seeming ly gluten-tolerant diet.

Despite an unremarkable clinical course, continued unrestricted gluten intake may predispose to the same sequelae as overt disease. 